\title{
Normal complement of motor units in asymptomatic familial (SOD1 mutation) amyotrophic lateral sclerosis carriers
}

\author{
A Aggarwal, G Nicholson
}

\begin{abstract}
Objective-To understand the mechanisms causing neuronal death in amyotrophic lateral sclerosis (ALS), an electrophysiological technique of motor unit number estimation (MUNE) was used to examine the pattern of motor neuron loss in amyotrophic lateral sclerosis. The aim was to determine whether gradual lifelong loss of motor units precedes clinical disease or whether sudden, catastrophic loss of motor units occurs at the onset of the disease.

Method-Using the statistical technique of motor unit number estimation, a cross sectional study was performed on a group of asymptomatic carriers of the $\mathrm{Cu}, \mathrm{Zn}$ superoxide dimutase 1 (SOD1) gene. MUNE results were compared with those from age and sex matched family controls who did not carry the SOD1 mutation. A total of 87 subjects ( 45 men and 42 women) with an age range from 16-73 years of age were studied.

Results-There was no detectable difference in the number of motor units in SOD1 mutation carriers compared with SOD1 negative family controls or population controls. Symptomatic subjects showed a definite loss of motor units. The test-retest reproducibility of this technique yielded an average difference between MUNE results on separate occasions on the same subject of $\pm 5 \%$.

Conclusion-The finding that presymptomatic SOD1 mutation carriers have a full complement of motor neurons indicates that mutation carriers must have normal survival of motor neurons until rapid and widespread cell death of these neurons occurs, coinciding with the onset of clinical features. This implies that symptomatic ALS is not the end result of a slow attrition of motor neurons.

(f Neurol Neurosurg Psychiatry 2001;71:478-481)
\end{abstract}

Neurobiology

Laboratory, ANZAC Research Institute,

Concord Hospital,

NSW, Australia 2139

A Aggarwal

G Nicholson

Correspondence to:

Dr A Aggarwal

arun@email.cs.nsw.gov.an

Received 19 June 2000 and in revised form

9 April 2001

Accepted 23 May 2001 that familial ALS is genetically heterogeneous and can be caused by mutations in more than one gene. The age of onset of ALS in SOD1 mutation carriers is variable, starting from the second decade. By the 9th decade, more than $90 \%$ of mutation carriers have developed the disease. ${ }^{2}$ Superoxide dismutase catalyses the superoxide radical $\left(\mathrm{O}^{2-}\right)$ into hydrogen peroxide and molecular oxygen. The SOD1 mutation was initially thought to reduce the neurons' protection from oxidation but weight of evidence now suggests that the mutated enzyme has a toxic gain of function. ${ }^{3}$

Subjects who are known SOD1 mutation carriers present a unique opportunity to study preclinical ALS. McComas ${ }^{4}$ showed that patients with substantial chronic denervation could maintain normal muscle twitch tension until the loss of about $70 \%-80 \%$ of motor units occurs, when collateral reinnervation was unable to provide further compensation. This indicates that considerable $(>70 \%)$ loss of motor neurons could occur before the onset of symptoms or weakness in patients with ALS. Early detection of motor neuron loss is important, if effective treatment for ALS is to be developed, as therapies aimed at preserving motor neurons may be more feasible than replacing lost motor neurons.

In ALS, there are two possible patterns of loss of motor neurons (fig 1). The first is a steady depletion of the neurons with age. In normal people, there is little decline in motor neuron number before the 7 th decade. After this, the number reduces by about $3 \%$ a year. ${ }^{5}$ If ALS was due to a slow attrition of motor neurons, it might be possible to detect preclinical motor neuron loss in carriers of the SOD1 mutation. The second pattern is maintenance of normal numbers of motor neurons until the disease process results in their sudden, rapid widespread cell death, corresponding with the development of symptoms. It has not been

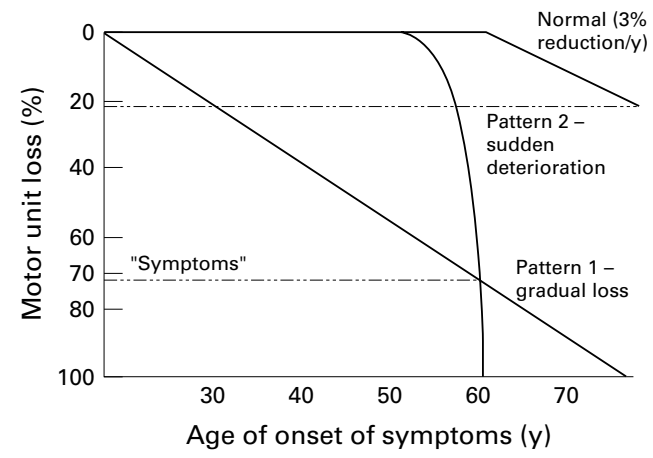

Figure 1 Diagram of possible patterns of loss of motor neurons in a person. 
possible to consider these questions until recently as presymptomatic diagnosis was impossible. Now, with the ability to identify SOD1 mutation carriers, presymptomatic subjects can be investigated. To determine if significant loss of motor neurons occurs years before the onset of disease, we performed a cross sectional study of at risk asymptomatic subjects (no neurological symptoms or signs as determined by a neurologist).

\section{Methods}

Family members of known SOD1 positive families were contacted and informed of this study. Eighty seven subjects (45 men and 42 women) gave informed consent. They participated without knowledge of their mutation status and on the understanding that this would not be disclosed to them. The neurologist performing the MUNE study also had no knowledge of their mutation status. The subjects were divided into four test groups (see below), with no statistically significant difference in age distribution, with a range of 16 to 73 years of age (table 1). The regional committee for ethics in medical research approved the study.

The four different point mutations present were glu100gly, ile113thr, val148gly, and val148ile. The MUNE results from asymptomatic SOD1 mutation carriers were grouped together. Although different mutations in SOD1 have different effects on the progression of the disease once symptoms occur, the different mutations do not influence the age of onset of symptoms. ${ }^{6}$

The study groups were:

(1) Twenty three population controls.

(2) Thirty four SOD1 negative (normal) family controls.

(3) Eighteen asymptomatic (preclinical) SOD1 mutation carriers (test group): five with point mutation in exon 4, codon 100, GAA to GGA, Glu to Gly; five with point mutation in exon 4, codon 113, ATT to ACT, Ile to Thr; four with point mutation in exon 5, codon 148, GTA to GGA, Val to Ile; four with point mutation in exon 5 , codon 148, GTA to GGA, Val to Gly.

(4) Twelve non-SOD1 mutation symptomatic patients with sporadic ALS (positive controls)

Motor unit numbers were routinely estimated in the right abductor pollicis brevis (APB) and extensor digitorum brevis (EDB) muscles. These muscles were used as the electrical activity can be recorded without interference, the muscle bellies are flat so the motor units are equidistant from the electrode, and in the case of EDB, there is only one end plate so

Table 1 Age distribution of subject groups

\begin{tabular}{lll}
\hline & $\begin{array}{l}\text { No of } \\
\text { Cases }\end{array}$ & $\begin{array}{l}\text { Mean age } \\
\text { (range) }\end{array}$ \\
\hline Population controls & 23 & $43(19-71)$ \\
SOD1 Negative family controls & 34 & $36(21-73)$ \\
SOD1 Mutation carriers & 18 & $42(16-68)$ \\
Sporadic ALS patients & 12 & $51(26-72)$ \\
\hline
\end{tabular}

all motor unit potentials summate. ${ }^{7}$ Self adhesive surface electrodes were used for recording, with G1 placed over the muscle belly and G2 over a bony prominence. The deep peroneal nerve was stimulated just above the ankle and the median nerve at the wrist with a surface stimulator, where the threshold of the nerve to electrical stimulation was lowest.

Although several MUNE techniques have been described, we used the statistical technique proposed by Daube. ${ }^{8}$ This was performed using proprietary software on a Nicolet Viking IV EMG machine. The low frequency filter was set at $2 \mathrm{~Hz}$ and the high frequency filter at $5 \mathrm{kHz}$. The gain for EDB was set at $2 \mathrm{mV} / \mathrm{div}$ and for APB studies at $5 \mathrm{mV} /$ div. The sweep speed was $2 \mathrm{~ms} /$ division. Motor unit numbers are obtained by dividing the size of the maximal compound muscle action potential (CMAP) by the size of the mean single motor unit potential (SMUP). The statistical MUNE technique derives the mean SMUP amplitude by analysis of the Poisson distribution of the variation in amplitudes after consecutive stimuli. An initial scan of the compound muscle action potential is performed using a series of 30 submaximal stimuli at $1 \mathrm{~Hz}$, increasing in equal increments to identify unusually large steps. On the basis of this scan, three or four $10 \%$ stimulus ranges are identified, according to an internal algorithm (fig 2). At each intensity, groups of 30 responses are captured at a rate of $3 \mathrm{~Hz}$, until the standard error of the MUNE SMUP size is less than $10 \%$.

All results were entered into a database and were analysed using a standard statistical software package. Motor unit estimates in carriers were compared with age and sex matched family controls without the SOD1 mutation, and patients with sporadic (non-SOD1) ALS. To determine whether groups had different numbers of motor units, an unpaired $t$ test was used. Although there were some outlying results, the distributions were not sufficiently skewed to contradict the use of the $t$ test.

To assess the test-retest reproducibility of the technique, MUNE was performed on 58 asymptomatic subjects and was repeated twice over a 1 year period. The difference between MUNE results from the first and second and first and third studies were divided by the MUNE of the first study and expressed as a percentage change. The results were analysed using Pearson correlation coefficients.

\section{Results}

There was no detectable difference in the number of motor units in asymptomatic SOD1 mutation carriers and their SOD1 negative family controls (APB $p>0.46$; EDB $p>0.95$ ), or population controls (APB, $p>0.70$; EDB, $\mathrm{p}>0.50$, table 2 and figs 3 and 4). Symptomatic patients with ALS had fewer motor units compared with all other groups $(\mathrm{p}<0001)$, with no overlap between symptomatic and asymptomatic subjects. There was no change in MUNE with age in any of the four groups. The study was powered with the ability to detect a difference in the group means of greater than 10 motor units.

The test-retest correlation of asymptomatic subjects was high (Pearson correlation 


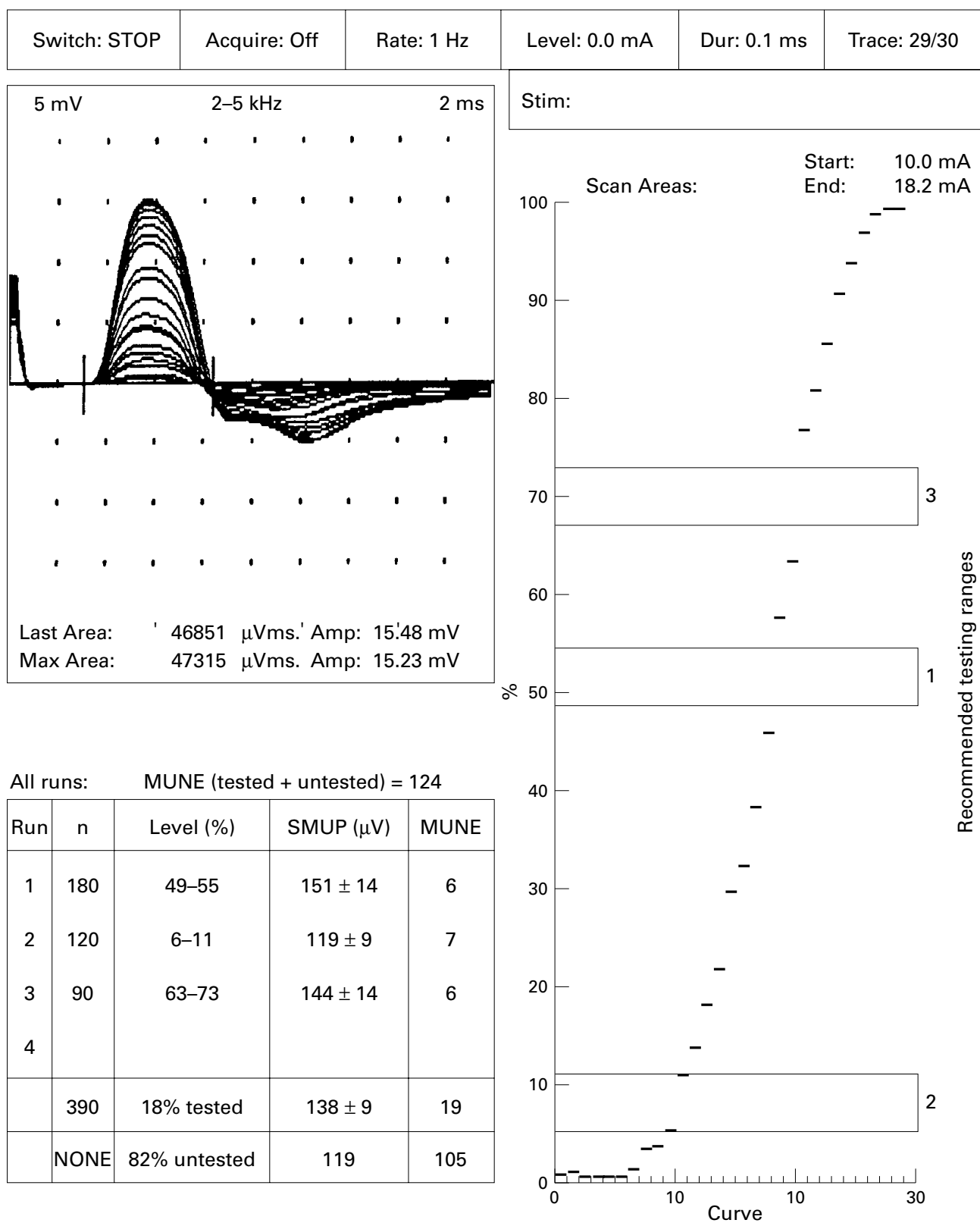

Figure 2 Stimulus-response curve from a series of 30 submaximal median nerve stimuli of increasing equal increments.

coefficient 0.93 for $\mathrm{APB}$ ). The mean difference between MUNE results on separate occasions on the same subject was $\pm 3.9 \%$ with a range of $0-11.7 \%$. For EDB, the Pearson correlation coefficient was 0.88 , with a mean difference between MUNE results on separate occasions on the same subject of $\pm 4.3 \%$, with a range of $0-10.6 \%$ (table 3 ). This compares favourably with published results. ${ }^{9}$

Table $2 A P B$ and EDB motor unit number estimates (MUNE number represents the mean $M U N E$ )

\begin{tabular}{llllll}
\hline & \multicolumn{2}{l}{ Abductor pollicis brevis } & & \multicolumn{2}{l}{ Extensor digitorum brevis } \\
\cline { 2 - 3 } $\begin{array}{l}\text { No of } \\
\text { cases }\end{array}$ & MUNE (range) & & $\begin{array}{l}\text { No of } \\
\text { cases }\end{array}$ & MUNE (range) \\
\hline Population controls & 23 & $148(115-254)$ & & 12 & $138(119-169)$ \\
SOD1 Negative family controls & 34 & $138(106-198)$ & & 32 & $134(107-180)$ \\
SOD1 Mutation carriers & 18 & $144(109-199)$ & & 14 & $136(111-187)$ \\
Sporadic ALS patients & 12 & $45(5-84)$ & & 9 & $70(8-82)$ \\
\hline
\end{tabular}

\section{Discussion}

Our aim was to determine whether subjects with the SOD1 mutation had a normal or reduced complement of functional motor neurons while asymptomatic. Felice ${ }^{10}$ identified MUNE as the most sensitive index for documenting changes in disease progression over time in patients with ALS. Azzouz et $a l^{11}$ also showed that in a transgenic mouse model which carried the human SOD1 gene clinical signs and pathology of disease correlated with a decrease in the number of motor units, as performed by MUNE. The results indicate that asymptomatic carriers of the SOD1 mutation have no significant difference in the number of motor neurons when compared with age and sex matched controls. This suggests that SOD1 mutation carriers have a normal complement of motor neurons while asymptomatic. The 


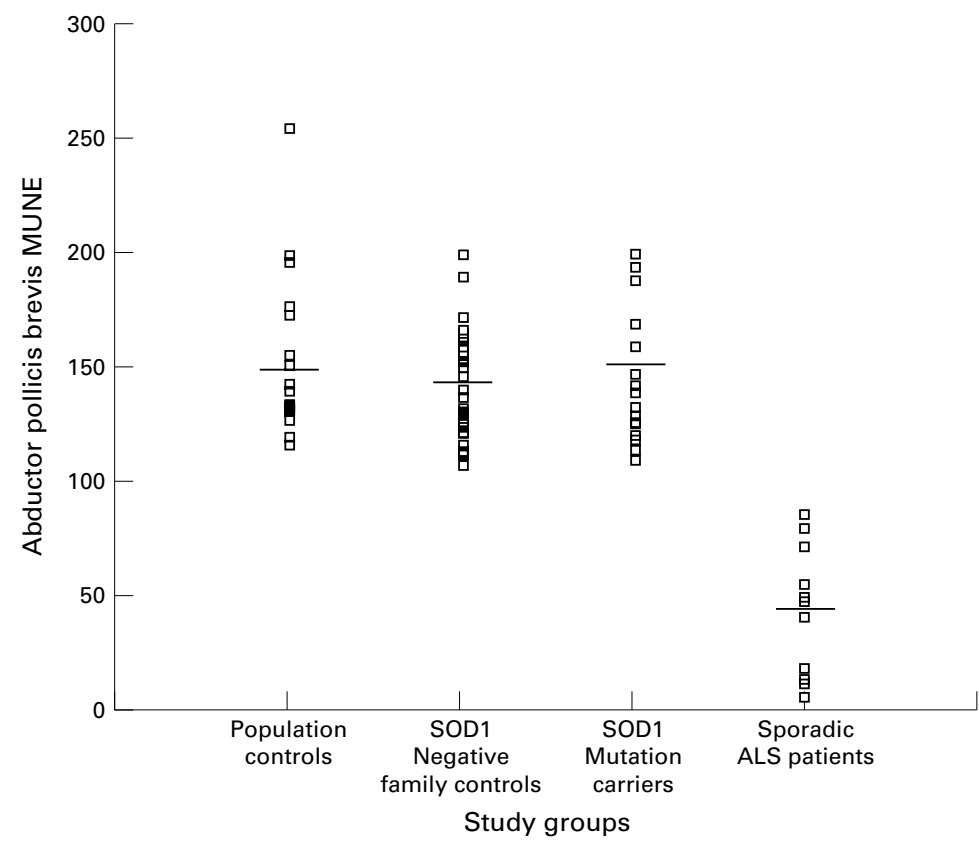

Figure 3 Abductor pollicis brevis motor unit number estimation subdivided into study groups (horizontal bar represents the mean MUNE).

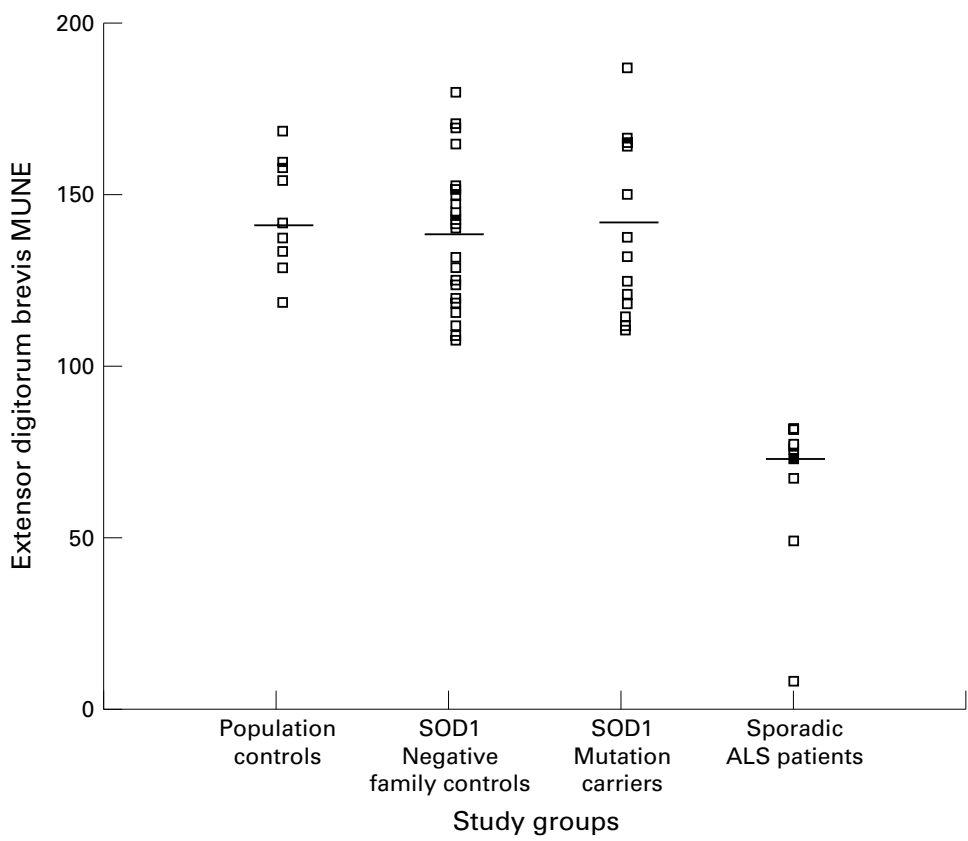

Figure 4 Extensor digitorum brevis motor unit number estimation subdivided into study groups (horizontal bar represents the mean MUNE).

development of symptoms must therefore be due to a relatively sudden, catastrophic loss of motor units.

Table 3 Reproducibility of $A P B$ and EDB motor unit number estimates in asymptomatic subjects on separate occasions over a 1 year period

\begin{tabular}{lll}
\hline & No of cases & $\begin{array}{l}\text { Mean } \\
\text { MUNE }\end{array}$ \\
\hline Abductor pollicis brevis 1 & 74 & 145.7 \\
Abductor pollicis brevis 2 & 58 & 140.1 \\
Abductor pollicis brevis 3 & 29 & 140.0 \\
Abductor pollicis brevis change & Range (0-11.7\%) & $3.9 \%$ \\
Extensor digitorum brevis 1 & 58 & 136.2 \\
Extensor digitorum brevis 2 & 51 & 132.5 \\
Extensor digitorum brevis 3 & 23 & 130.4 \\
Extensor digitorum brevis change & Range (0-10.6\%) & $4.3 \%$ \\
\hline
\end{tabular}

This study forms part of a longitudinal study to follow the rate of motor neuron loss in individual asymptomatic SOD1 mutation carriers. Our results indicate that the reproducibility of this technique is good, and individual results can be used as a baseline for serial MUNE studies. Due to the wide variability of motor unit numbers in normal subjects, a large sample size is needed to provide sufficient statistical power to detect small differences between the means for asymptomatic carriers and the control group. This small number would not be detectable by a change in the mean MUNE because most asymptomatic subjects would dilute this change. Following up individual SOD1 mutation carriers over time will help to determine whether a slight loss of motor neurons is occurring, which cannot be detected in cross sectional studies, due to these interindividual variations.

Regular follow up in these subjects may help to identify those who may benefit from early institution of an active management programme to improve their quality of life. If it is possible to detect a gradual loss of motor neurons over time in asymptomatic subjects, this may lead to early diagnosis, creating an opportunity for new approaches for preventing the progression of this devastating disease.

This study suggests that significant gradual preclinical loss of motor neurons is not occurring in asymptomatic SOD1 mutation carriers. Therefore, we suggest that sudden and widespread motor neuron death must occur at the time of development of the clinical symptoms, rather than lifelong motor neuron loss. This finding is an important contribution to the current understanding of the pathogenesis of amyotrophic lateral sclerosis.

This work was carried out as part of a $\mathrm{PhD}$ project at the University of Sydney. We acknowledge the Motor Neurone Disease Association of New South Wales (Northern Region), ANZAC Health and Medical Research Foundation, Motor Neuron Disease Research Institute of Australia Inc, and the Nerve Research Foundation for their financial support for this study. We thank Dr G Herkes, Department of Neurology, Royal North Shore Hospital and Ms Joan Petch, Prince Charles Hospital, Brisbane for their support.

1 Rosen, DR, Siddique T, Patterson D, et al. Mutations in Cu, $\mathrm{Zn}$ superoxide dismutase gene are associated with familial amyotrophic lateral sclerosis. Nature 1993;362:59-62.

2 de Belleroche J, Orrell R, King A. Familial amyotrophic lateral sclerosis/motor neurone disease (FALS): a review of current developments. F Med Genet 1995;32:841-7.

3 Siddique T, Deng HX. Genetics of amyotrophic lateral sclerosis. Human Mol Genet 1996;5:1465-70.

4 McComas AJ. Functional compensation in partially denervated muscles. F Neurol Neurosurg Psychiatry 1971;34:45360.

5 Campbell MJ, McComas AJ, Petito F. Physiological changes in ageing muscles. $\mathcal{F}$ Neurol Neurosurg Psychiatry 1973;36: 174-82.

6 Andersen PM, Nilsson P, Keranen M-L, et al. Phenotypic heterogeneity in motor neuron disease patients in CuZnsuperoxide dimutase mutations in Scandinavia. Brain 1997;120:1723-37.

7 Sica REP, McComas AJ, Upton ARM, et al. Motor unit estimations in small muscles of the hand. F Neurol Neurosurg Psychiatry 1974;37:55-67.

8 Daube JR. Estimating the number of motor units in a muscle. F Clin Neurophysiol 1995;12:585-94.

9 Olney RK, Yuen EC, Engstrom JW. Statistical motor unit number estimation: reproducibility and sources of error in patients with amyotrophic lateral sclerosis. Muscle Nerve 2000;23:193-7.

10 Felice KJ. A longitudinal study comparing thenar motor unit number estimation to other quantitative tests in patients with amyotrophic lateral sclerosis. Muscle Nerve $1997 ; 20: 179-85$.

11 Azzouz M, Leclerc N, Gurney M, et al. Progressive motor neuron impairment in an animal model of familial amyotrophic lateral sclerosis. Muscle Nerve 1997;20:45-51. 